\title{
CORRELATION BETWEEN WONG-BAKER FACES PAIN SCALE AND SALIVARY ALPHA-AMYLASE LEVEL IN CHILDREN AGED 6-11 YEARS
}

\author{
HASTIN DIAN ANGGRAENI ${ }^{1}$, MARGARETHA SUHARSINI ${ }^{2 *}$, IKE SITI INDIARTI ${ }^{1}$, FARAGHEA YUMASDHIKA ${ }^{1}$ \\ ${ }^{1}$ Pediatric Dentistry Residency Program, Faculty of Dentistry, Universitas Indonesia, Indonesia. ${ }^{2}$ Department of Pediatric Dentistry, \\ Faculty of Dentistry, Universitas Indonesia, Indonesia. Email: margarethasuharsini@gmail.com
}

Received: 16 September 2017, Revised and Accepted: 3 October 2017

\section{ABSTRACT}

Objective: Several studies have tried to objectively assess pain measurements. The Wong-Baker Faces Pain Scale (WBFPS) is an instrument that is commonly used to assess pain intensity in children.

This study aimed to analyze the correlation between the WBFPS and salivary alpha-amylase(SAA) level during a tooth extraction procedure with a local anesthetic injection in children aged 6-11 years

Methods: Twenty-five children aged 6-11 years who were to undergo the extraction of a primary tooth at the Dental and Oral Educational Hospital, Faculty of Dentistry Universitas Indonesia, were enrolled in this study. From all children, saliva was collected using a disposable saliva strip shortly after local anesthetic injection, and the SAA activity was then determined using a portable Nipro Cocoro Meter device. The WBFPS was measured at the same time. The correlation between the WBFPS and the SAA level was analyzed using Spearman's correlation test. The statistically significant level was set at $\mathrm{p} \leq 0.05$.

Results: There was a significant correlation between the WBFPS and SAA level $(\mathrm{p}=0.002, \mathrm{r}=0.581)$.

Conclusion: Our data suggest that the SAA level might be a good index for objective pain intensity assessment.

Keywords: Pain, Salivary alpha-amylase, Children.

(C) 2017 The Authors. Published by Innovare Academic Sciences Pvt Ltd. This is an open access article under the CC BY license (http://creativecommons. org/licenses/by/4. 0/) DOI: http://dx.doi.org/10.22159/ijap.2017.v9s2.34

\section{INTRODUCTION}

Pain has sensory, emotional, cognitive, and behavioral components that are interrelated with environmental, developmental, sociocultural, and contextual factors. It is a complex multidimensional concept that can vary in quality, intensity, duration, location, and unpleasantness [1]

Finding a gold standard for the objective assessment of pain in young children is a challenging and critical task for health professionals. An accurate and reliable measurement of pain is necessary both for diagnostic purposes and for evaluating pain behavior [1]. Pain can be assessed through self-report measures, behavioral measures (e.g., facial expression and behavioral rating), and physiological measures (e.g., heart rate, sweating, and EEG). The choice of the proper instrument depends on the nature of the painful stimulus (chronic or acute), age of the child, and his/her communication capabilities [2].

Most children aged 5 years and older, and even many 3- and 4-year-olds, can provide a meaningful self-report of pain if age-appropriate tools are used. Self-report measurement tools include visual analog scales (VAS), numerical rating scales, faces pain scales, color analog scales (CAS), and pieces-of-hurt (poker chip) scale. Faces pain scales include the Faces Pain Scale (FPS), Faces Pain Scale-Revised (FPS-R), Oucher Pain Scale, and Wong-Baker Faces Pain Scale (WBFPS). Tomlinson compared these four measurements and found that children preferred the WBFPS [3]. Salivary alpha-amylase (SAA) concentrations have been used to determine pain-induced stress levels. Shirasaki found good correlation between scales of pain intensity and SAA. This is because painful stimuli activate the sympathetic-adrenal medullary (SAM) system and hypothalamic-pituitary-adrenal (HPA) axis [4]. Some children need their primary teeth to be extracted under local anesthetic injection during the exfoliation period. Nevertheless, relatively few studies have focused on pain evaluation in children during the injection procedure. Given the limited number of studies performed to evaluate the relationship of alpha-amylases, especially SAA, with pain, the present study aims to evaluate the relationship between SAA and pain intensity in children due to local anesthetic injection during the tooth extraction procedure.

\section{METHODS}

Twenty-five children ( 9 males and 16 females) aged 6-11 years who were to undergo the extraction of a primary tooth at the Dental and Oral Educational Hospital, Faculty of Dentistry, Universitas Indonesia, were enrolled to participate in this study. Informed consent was obtained from the children parents or caregivers before taking samples. The protocol and patients informed consent forms were approved by the Ethics Committee of Faculty of Dentistry, Universitas Indonesia.

After examination and diagnosis determination, the patients received a topical anesthetic with Ultradent $20 \%$ benzocaine gel at the mucobuccal fold area closest to the extracted tooth for 1 min. Then, a local anesthetic with articaine HCL $4 \%$ with epinephrine 1:100,000 (Septocaine-Septodont) was injected through a Morita 30G dental needle using a Paroject intraligamental syringe. The WBFPS and SAA were measured shortly after local anesthetic injection.

Saliva was collected sublingually using a disposable test strip consisting of saliva-collecting paper and reagent paper. The test strips consist of an outer sleeve, a sheet, a collecting paper, and an amylase reagent paper. The strip was inserted into a portable SAA activity monitor (Nipro Cocoro meter, Japan) consisting of a salivary transcription device and an optical analyzer. On closing the lever, saliva is transferred from the test strip to the reagent paper. Concurrently, a timer begins when 
the lever is closed, and it rings after saliva transfer is complete. This process takes approximately 10 seconds. After the alarm rings, the strip is pulled from the sleeve. After 20 seconds, an SAA reading is available. The total measurement time is approximately 60 s, including 30 s for the collection of saliva, 10 s for the transcription of saliva from the collecting paper to the reagent paper, and 20s for the measurement of the color depth. The WBFPS was shown to all children, and they were asked to indicate the smiling face that is closest to their mood and their feelings at that moment.

In this way, we obtained information directly from children about their perception of pain. The correlation between the WBFPS and the SAA level was assessed using Spearman's correlation test; $\mathrm{p}<0.05$ was considered statistically significant.

\section{RESULTS}

In this study, $36 \%$ of the participants were male, and $67 \%$ were female. The children age distribution was as follows: 6 years $(4 \%), 7$ years (16\%), 8 years (28\%), 9 years (16\%), 10 years $(24 \%)$, and 11 years $(12 \%)$. The pain intensities reported were from 2 to 6 , and the SAA level reported was from 18 to $161 \mathrm{unit} / \mathrm{ml}$. Table 1 summarized that the difference in SAA activity between the males and the females was not significant (Mann-Whitney U-test, $p=0.207$ ), and neither was the difference in pain severities (Mann-Whitney U-test, $p=0.934$ ).

Both SAA level and pain did not correlate significantly with age $\left(r_{1}=0.148, r_{2}=0.095, p_{1}=0.48, p_{2}=0.652\right.$, Table 2$)$.

\section{DISCUSSION}

This study showed that the SAA level was in accordance with the level of pain reported by the children during the infiltration injection of a local anesthetic. These results are similar to those reported previously by Shirasaki and Motamayel [4,5]. It has been reported that psychological stress activates the SAM system [6]. This, in turn, increases plasma norepinephrine, and this mechanism increases SAA secretion. Pain is considered a stressful agent, and therefore, painful stimuli will activate this system [7]. Through this mechanism, SAA may serve as a marker of pain.

In this study, participants reported pain with intensities of 2 to 6 on the WBFPS. There is a statistically small but insignificant correlation between the pain intensity and the children age. A previous study has demonstrated that most children aged 5 years and older, and even many 3- and 4-year-olds, can provide a meaningful self-report of pain if age-appropriate tools are used [3].

The SAA level varies from 18-161 unit/ml. There is a statistically small correlation between the SAA level and the children age. No difference is seen in alpha-amylase activity between males and females. The result of a previous study showed no difference between SAA levels by gender [8]. Previous studies also found that secretion of alpha-amylase in the saliva increased from 2 to 6 months of age and that the SAA level of children aged 6-12 months was relatively equivalent to the adult level [9].

\section{CONCLUSION}

According to our results, the SAA level was significantly correlated with the pain levels assessed using the WBFPS. This result suggests that the SAA level could serve as a new objective and non-invasive biomarker to assess pain perception in children during the injection of local anesthesia.
Table 1: Mean and SD of Wong-Baker score and SAA level of participants

\begin{tabular}{llll}
\hline & $\mathbf{n}$ & Wong-Baker score & SAA level (unit/mL) \\
\hline Male & 9 & $3.33 \pm 1.414$ & $68.67 \pm 48.941$ \\
Female & 16 & $3.25 \pm 1.238$ & $47.00 \pm 30.930$ \\
Total & 25 & $3.28 \pm 1.275$ & $54.80 \pm 38.846$ \\
p value & & 0.934 & 0.207 \\
\hline
\end{tabular}

*Mann-Whitney U-test. SD: Standard deviation, SAA: Salivary alpha-amylase

Table 2: Spearman's correlation coefficient between Wong-Baker score and SAA level

\begin{tabular}{lll}
\hline & Age & SAA level \\
\hline Wong-Baker & $\mathrm{r}=0.095$ & $\mathrm{r}=0.581$ \\
SAA level & $\mathrm{p}=0.652$ & $\mathrm{p}=0.002$ \\
& $\mathrm{r}=0.148$ & \\
\hline
\end{tabular}

Spearman's correlation analysis showed a significant correlation between the WBFPS and SAA level ( $\mathrm{p}=0.002, \mathrm{r}=0.581)$. SAA: Salivary alpha-amylase, WBFPS: Wong-Baker Faces Pain Scale

\section{ACKNOWLEDGMENT}

The authors thank to the PITTA Grant from Directorate of Research and Community Service Universitas Indonesia, for financially supporting this research. We also would like to thank the Wong-Baker Foundation for providing the pain scale in the Indonesian language.

The publication of this manuscript is supported by Universitas Indonesia.

\section{REFERENCES}

1. American Academy of Pediatric. The assesment and management of acute pain in infants, children, and adolescent. Pediatrics 2001;108:3

2. Versloot J, Veerkamp JS, Hoogstraten J. Pain behaviour and distress in children during two sequential dental visits: Comparing a computerised anaesthesia delivery system and a traditional syringe. Br Dent J 2008;205:E2.

3. Tomlinson D, von Baeyer CL, Stinson JN, Sung L. A systematic review of faces scales for the self-report of pain intensity in children. Pediatrics 2010;126:e1168-98.

4. Shirasaki S, Fujii H, Takahashi M, Sato T, Ebina M, Noto Y, et al. Correlation between salivary alpha-amylase activity and pain scale in patients with chronic pain. Reg Anesth Pain Med 2007;32:120-3.

5. Ahmadi-Motamayel F, Shahriari S, Goodarzi MT, Moghimbeigi A, Jazaeri M, Babaei P, et al. The relationship between the level of salivary alpha amylase activity and pain severity in patients with symptomatic irreversible pulpitis. Restor Dent Endod 2013;38:141-5.

6. Strahler J, Mueller A, Rosenloecher F, Kirschbaum C, Rohleder N. Salivary alpha-amylase stress reactivity across different age groups. Psychophysiology 2010;47:587-95

7. Campos MJ, Raposo NR, Ferreira AP, Vitral RW. Salivary alphaamylase activity: A possible indicator of pain-induced stress in orthodontic patients. Pain Med 2011;12:1162-6.

8. Dezan CC, Nicolau J, Souza DN, Walter LR. Flow rate, amylase activity, and protein and sialic acid concentrations of saliva from children aged 18, 30 and 42 months attending a baby clinic. Arch Oral Biol 2002;47:423-7

9. Davis EP, Granger DA. Developmental differences in infant salivary alpha-amylase and cortisol responses to stress. Psychoneuroendocrinology 2009;34:795-804 